médecine/sciences 1986; $2: 130-6$

\title{
L'imagerie par résonance magnétique se substituera-t-elle à la tomodensitométrie?
}

L'imagerie par résonance magnétique tend déjà à remplacer la tomodensitométrie dans l'exploration de certaines lésions du système nerveux central et de la moelle. Une substitution partielle peut être envisagée dans d'autres domaines.

\section{Philippe Grenier \\ Professeur à la faculté Xavier Bichat, Université Paris VII}

\section{Marie-Thérèse Desbleds Attaché-consultant}

\section{Jean-Charles Guilbeau Chef de Clinique-assistant}

\section{ADRESSE}

P. Grenier, MT. Desbleds, JC. Guilbeau : Service de radiologie, hôpital Beaujon, roo, boulevard du Général-Leclerc, 921 I 8 Clichy Cedex.
- imagerie par résonance magnétique (IRM) est une technique coûteuse, sophistiquée, mais hautement utile qui arrive aujourd'hui sur la scène de la pratique clinique médicale. Les enjeux économiques sont considérables et l'acquisition d'un appareillage IRM par une structure hospitalière traditionnelle reste extrêmement difficile. Dès lors, une question s'impose à tous ceux qui ont pour charge de planifier l'acquisition des équipements lourds nécessaires à l'imagerie médicale à l'échelle d'un . centre hospitalier régional ou d'un pays : ia substitution de la tomodensitométrie (TDM) par l'IRM est-elle possible? Si oui, dans quelle proportion et quand surviendra-t-elle?

Il est aujourd'hui difficile de répondre avec certitude et précision à une telle question. Des hypothèses établies sur des perspectives faites à dix ans ont pu être émises par des économistes dans quelques pays industrialisés, mais ces hypothèses sont parf ois contradictoires et ne reposent actuellement sur aucune vérité objective $[1,2]$. En effet, les évaluations comparatives coûtefficacité entre la TDM et l'IRM sont loin d'avoir atteint leur maturité. La raison en est simple, car l'évaluation économique de toute technique d'imagerie ne devient possible que lorsque les possibilités diagnostiques de cette technique ont été préalablement évaluées dans tous les domaines de la pathologie. Or, l'étape clinique de l'évaluation de l'IRM n'en est qu'à sa phase préliminaire. Les travaux publiés concernent essentiellement des études descriptives et les études comparatives TDM-IRM restent encore très limitées et peu rigoureuses sur le plan méthodologique. Des évaluations prospectives et comparatives des performances diagnostiques des deux techniques nécessitent des protocoles cliniques lourds et prolongés. De nombreuses études de ce type sont aujourd'hui engagées en Amérique du Nord, au Japon et en Europe, mais il y a peu de chance de pouvoir en exploiter les résultats avant une période allant de deux à cinq ans. L'évolution constante de la technologie ne facilite pas l'exploitation rapide des résultats de telles ćvaluations cliniques. Une étude est renduc bien souvent inutile avant d'avoir atteint son terme, du fait de l'apparition continue d'améliorations techniques qui interfèrent avec le protocole d'examen.

Malgré ces difficultés et ces incertitudes, il apparaît qu'une certaine substitution de la TDM par l'IRM s'imposera à terme. Ce qui reste encore mystérieux, c'est dans quelle proportion, dans quel domaine de la pathologie et à quelle vitesse se fera cette substitution. Des contraintes d'ordre économique et politique propres à chaque pays influent certainement sur la dynamique de la substitution, mais ces considérations dépassant à l'évidence le cadre de cette revue, nous nous contenterons de faire ici un " état de l'art " à partir des données scientifiques recueillies dans la littérature. Les données rassemblées permettront de préjuger à ce jour d'une certaine substitution. Elles mériteront d'être 
affinées périodiquement au cours des mois et des années à venir. L'analyse et la synthèse des résultats seront abordées séparément selon les grands domaines d'application de l'IRM.

\section{En neuroradiologie}

Les problèmes diffèrent selon qu'il s'agit d'examens cranio-encéphaliques ou d'examens médullaires. Dans l'exploration cranio-encéphalique, l'examen TDM a bouleversé depuis douze ans les méthodes d'investigations neuroradiologiques. Permettant la visualisation directe du parenchyme et des ventricules cérébraux pour une irradiation acceptable et sans risques particuliers, si ce n'est ceux imputables au produit de contraste iodé, la TDM s'est imposée comme le premier examen radiologique demandé pour tout malade présentant un, ou des signes neurologiques. Associée au contexte clinique, la TDM permet le diagnostic dans la majorité des cas, ou l'oriente fortement. Dès lors, l'encéphalographic gazeuse n'est plus réalisée et c'est là un exemple de substitution totale. Plus partielle, mais non moins importante, est la substitution des examens angiographiques, dont seules persistent quelques indications pré-opératoires et le bilan des lésions artérielles et veineuses. Néanmoins, dans un certain nombre de cas, la TDM reste insuffisante, soit par artéfacts inhérents à la technique, soit du fait d'examens faussement négatifs, soit d'une difficulté à localiser anatomiquement une lésion, soit de l'impossibilité à reconnaître la nature de la lésion objectivée. De ce fait, l'IRM a soulevé l'enthousiasme dès son apparition, permettant une excellente étude morphologique aussi bien à l'étage sus-tentoriel qu'à l'étage sous-tentoriel (figure I). Les contrastes obtenus entre le liquide céphalo-rachidien (LCR) et le parenchyme cérébral, entre la substance blanche et la substance grise sont supérieurs à ceux vus en TDM. L'IRM permet des coupes dans plusieurs directions de l'espace, sans avoir à mobiliser le patient ( $f i g u-$ re 2). Elle s'avère être une technique plus sensible que la TDM dans la détection des anomalies chez des $\mathrm{m} / \mathrm{s} n^{\circ} 3$ vol. 2 mars 86

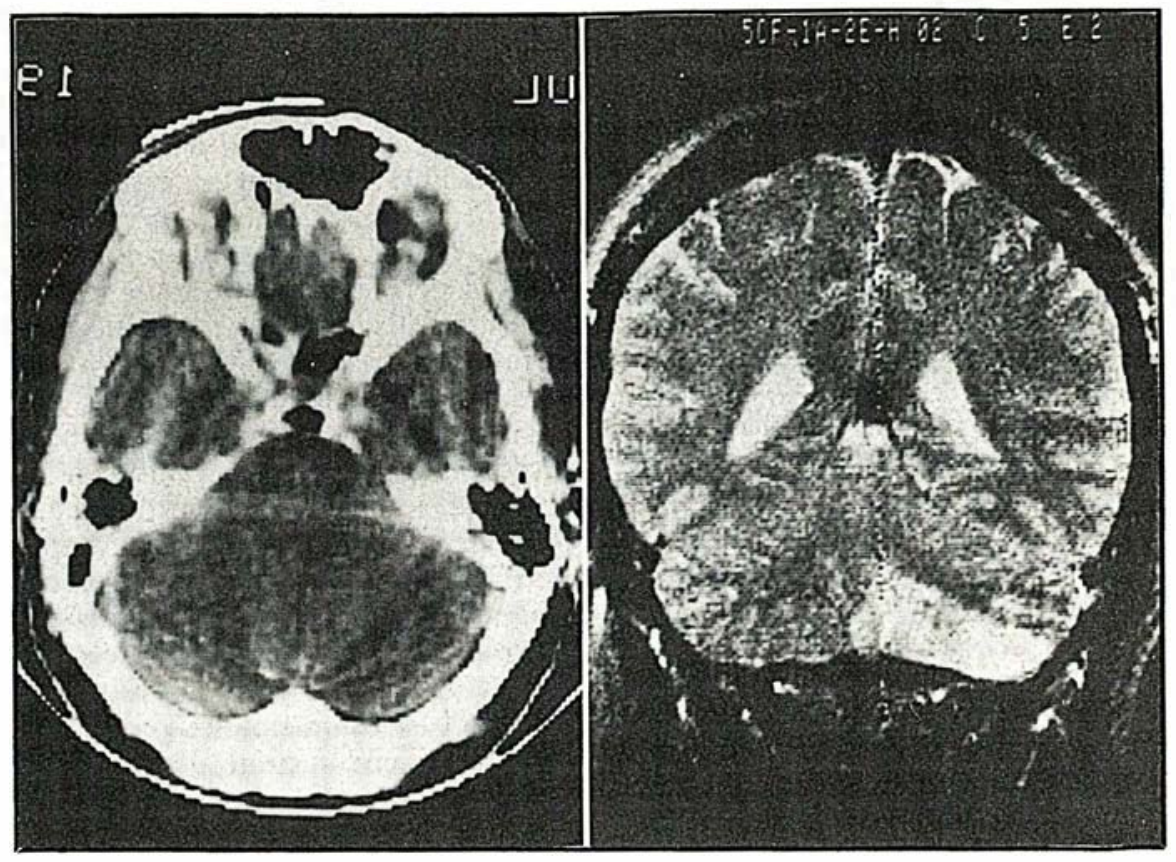

Figure I. Accident ischémique cérébelleux gauche.

A gauche, tomodensitométrie : effet de masse visible par l'oblitération des citernes et déviation du quatrième ventricule.

A droite, IRM : visualisation de la lésion ischémique responsable de leffet de masse.

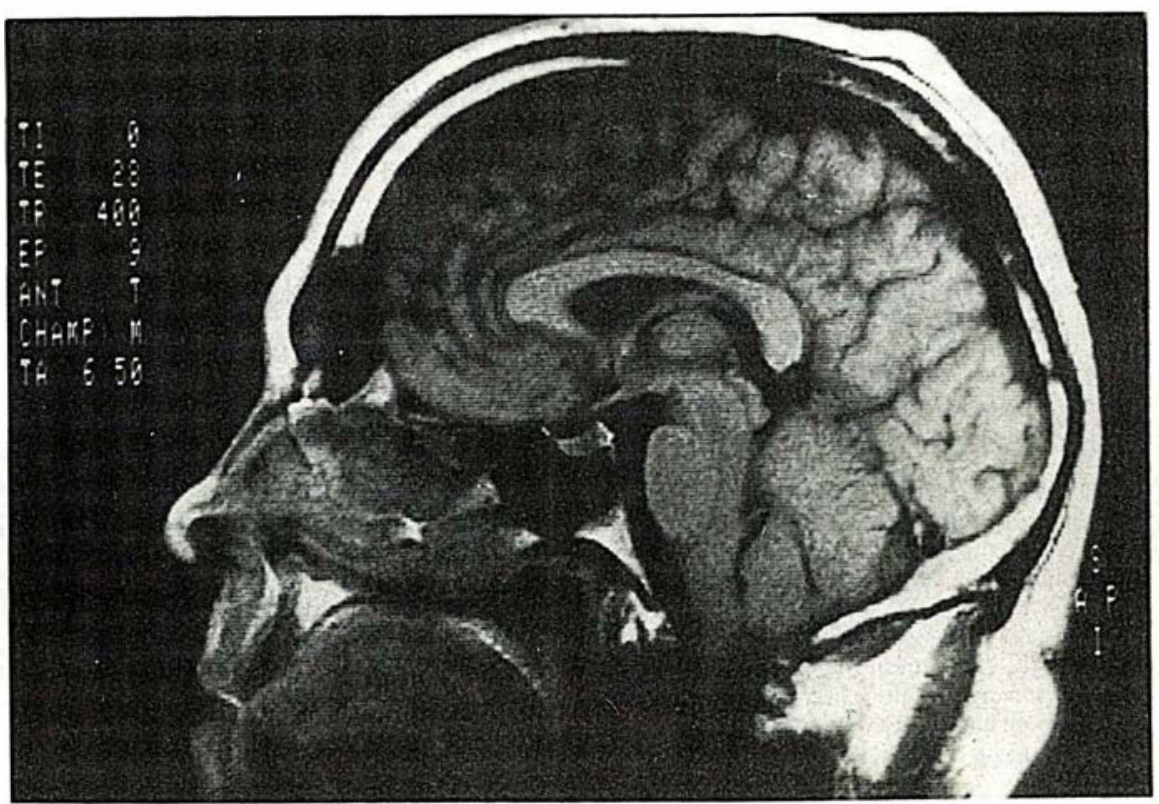

Figure 2. Coupe sagittale médiane de l'encéphale.

Excellente définition des structures médianes sus et sous-tentorielles. 


\section{RÉFÉRENCES}

I. Evens RG, Jost RG, Evens Jr RG. Economic and utilization analysis of magnetic resonance imaging units in the United States in $1985 . A 7 R$ $1985 ; 145: 393-8$.

2. Murphy WA. How does magnetic resonance compare with computed tomography? Radiology 1984; $152: 235-6$.

3. Bradley WG, Waluch V, Yadley RA, Wycoff RR. Comparison of computed tomography and magnetic resonance in 400 patients with suspected diseasc of the brain and cervical spinal cord. $R a-$ diology 1984; 152 : 695-702.

4. Sipponen JT, Kaste M, Ketonen L, et al. Serial nuclear magnetic resonance (NMR) imaging in patients with cerebral infarction. 7 Comput Assist Tomogr 1983; 7 : 585-9.

5. Gomori JM, Grossman RI, Goldberg RI. Intracranial hematomas: imaging by high-field magnetic resonance. Radiology 1985; $157: 87-93$.

6. Kucharczyk W, Lemme-Pleghos I, Uske A, et al. Intracranial vascular malformations: magnetic resonance and computed tomography imaging. Radiology 1985; 156: 383-9.

7. Rinck PA, Meindl S, Higer HP, et al. Brain tumors: detection and typing by use of CPMG sequences and in vivo $\mathrm{T}_{2}$ measurements. Radiology 1985; $157:$ 103-6.

8. Felix R, Schorner W, Laniado M, et al. Brain tumors: magnetic resonance imaging with Gadolinium-DPTA. Radiology 1985; $156: 68 \mathrm{I}-8$.

9. Le Bihan D, Breton E, Lallemand D, et al. Diff usion magnetic resonance imaging: neurological results. Communication for the 7 Ith Assembly of RSNA, Chicago, 1985

10. Brant-Zawadzki M, Davis PL, Crooks LE, et al. NMR demonstration of cerebral abnormalities: comparison with computed tomography. $A 7 R$ 1983; 140: 847-54.

II. Bilaniuk LT, Schenck JF, Zimmerman RA Ocular and orbital lesions: surface coil magnetic resonance imaging. Radiology $1985 ;$ i 56 : 669-74.

12. Gebarski SS, Maynard FW, Gabrielsen TO, et al. Post-traumatic progressive myelopathy. $R a$ diology $1985 ; 157: 379-85$.

13. Levitt RG, Glazer HS, Roper CL, et al. Magnetic resonance imaging of mediastinal and hilar masses: comparison with computed tomography. $A 7 R$ 1985; $145: 9-14$.

14. Webb WR, Jensen BG, Sollitto R, et al. Bronchogenic carcinoma: staging with magnetic resonance compared with staging with computed tomography and surgery. Radiology $1985 ; 156$ II 7-24.

15. Ross JS, O'Donovan PB, Novoa R, et al. Magnetic resonance of the chest initial experience with imaging and in vivo $T_{1}$ and $T_{2}$ calculations. Radiology 1984; 152: 95-101.

16. Muller NL, Gamsu G, Webb WR. Pulmonary nodules: detection using magnetic resonance and computed tomography. Radiology $1985 ; 155$ : 687-90. patients symptomatiques. Dans un travail récent [3] portant sur 400 patients suspects d'atteinte neurologique centrale, des comparaisons TDM-IRM ont pu être faites. Parmi les 325 patients présentant une réelle lésion neurologique, l'IRM permettait à elle seule le diagnostic dans 93 cas et était plus contributive que la TDM dans 68 autres cas. Par ailleurs, chez 125 patients, les lésions étaient aussi bien étudiées par les deux méthodes et chez 32, les lésions étaient plus facilement reconnues sur la TDM que sur l'IRM. Les lésions mieux étudiées par l'IRM correspondaient surtout à des lésions de petite taille ou à des lésions de la fosse postérieure. Les lésions mieux vues par la TDM étaient surtout des tumeurs calcifiées, des encéphalites ou des méningiomes. Les résultats de cette étude, bien que favorables à l'IRM, témoignent plus d'une certaine complémentarité que d'une véritable substitution.

D'autres études comparatives ont été faites dans des cadres pathologiques plus restreints et orientées sur les problèmes de spécificité propre à chacune des deux méthodes. Dans les maladies vasculaires, les lésions ischémiques parenchymateuses précoces sont mieux décelées par l'IRM que par la TDM mais le manque de spécificité des anomalies morphologiques constatées persiste [4]. Les hématomes intra-crâniens ne sont bien vus en IRM qu'après une semaine d'évolution. Les hématomes récents ne sont pas repérables en imagerie utilisant des valeurs de champ magnétique inférieures à 1 Tesla [5].

Dans le diagnostic des malformations artérioveineuses, l'IRM apparaît aussi contributive que la TDM et semble plus précise dans la détection des hémorragies associées [6].

En pathologie tumorale, la sensibilité de l'IRM est supérieure à celle de la TDM, mais la caractérisation des masses tumorales détectées reste un échec [7]. La spécificité de l'IRM n'est pas supérieure dans ce domaine car les paramètres de relaxation qui interviennent dans le contraste des images ne sont pas suffisants pour identifier la nature tissulaire et histologique de la tumeur. Les calcifications si bien re- pérées en TDM, échappent le plus souvent à l'IRM. La distinction entre la tumeur et l'œdème qui l'accompagne, si bien vue en TDM après injection de produit de contraste, reste mal visible sur l'IRM (figure 3). Pour pallier ces insuffisances, les recherches actuelles s'orientent vers l'utilisation de produits de contraste paramagnétiques [8], l'optimisation des séquences et l'exploitation d'autres paramètres du contraste comme la diffusion ou la perfusion [9].

Enfin, l'IRM trouve une place privilégiée dans d'autres domaines, où elle impose sa supériorité sur la TDM, comme le diagnostic des hydrocéphalies communicantes ou celui des maladies démyélinisantes, en particulier la sclérose en plaques [ı]. Les explorations de l'œil, de l'orbite et de l'oreille deviennent possibles en IRM grâce à l'utilisation des antennes de surface[II]. Les examens IRM restent encore de durée assez longue, rendant l'exploration difficilement réalisable chez les patients agités ou peu conscients. Des problèmes de surveillance, non encore réglés rendent l'IRM encore non réalisable chez les sujets comateux ou les patients nécessitant une assistance respiratoire. Si l'on exclut le cas des lésions de la fosse postérieure, du tronc cérébral ou de l'axe hypothalamo-hypophysaire qui bénéficient largement de l'apport de l'IRM, une substitution TDMIRM ne peut guère être envisagée à court terme.

L'exploration de la moelle est certainement un des domaines où l'IRM s'impose avec le plus de certitude. L'imagerie sagittale se prête parfaitement à l'exploration du canal médullaire. Le contraste spontané obtenu entre la moelle et le LCR est suffisant pour analyser la plupart des processus expansifs extra-duraux, sous-duraux et intramédullaires. L'utilisation d'antennes de surface améliore la résolution spatiale et facilite l'étude des petites lésions. L'IRM est largement supérieure à la TDM dont le protocole d'exploration reste limité à l'imagerie axiale transverse et nécessite l'injection intra-thécale de produit de contraste. Fiable et parfaitement non invasive, l'IRM va vite devenir la technique de choix pour l'explo- 

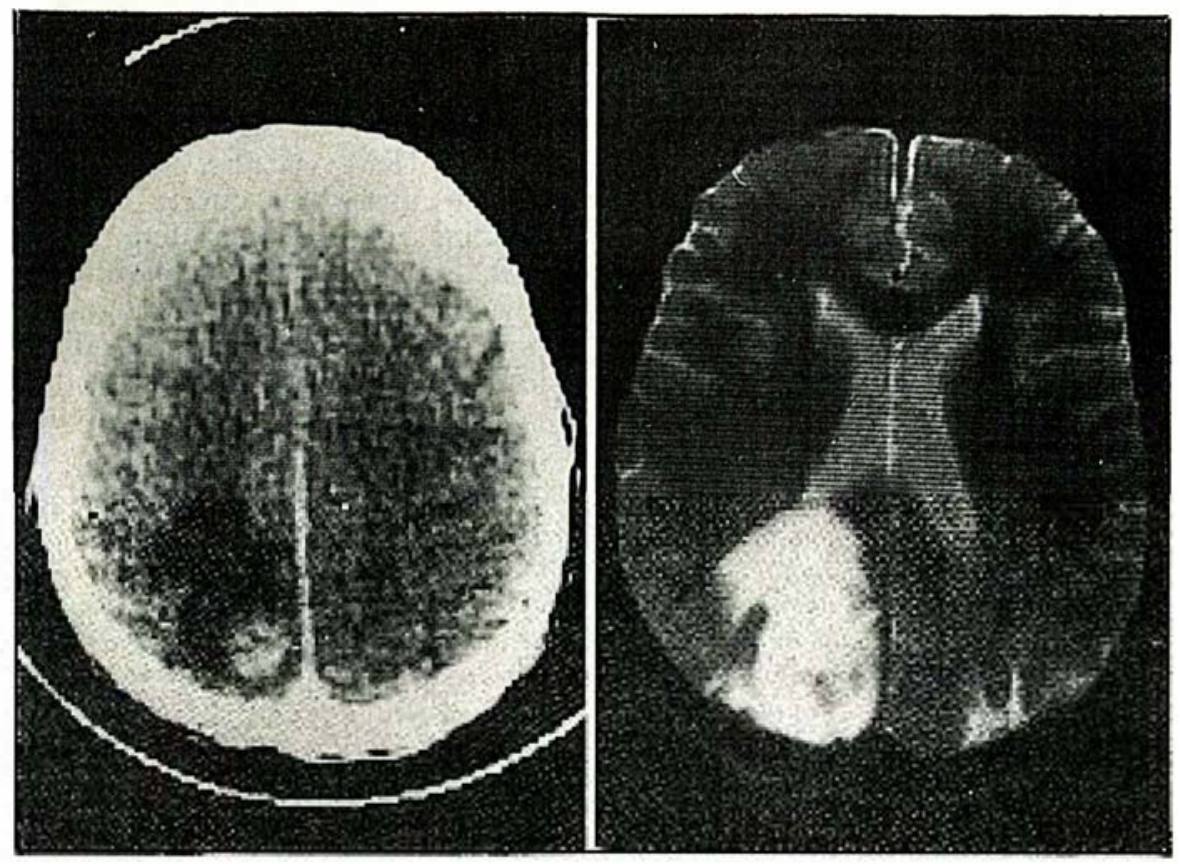

Figure 3. Métastase pariétale droite. A gauche, tomodensitométrie : le contraste iodé permet de délimiter la tumeur au sein de l'adème.

A droite, IRM : la distinction entre l'adème et la tumeur est impassible.

ration des tumeurs de la moclle, des myélopathies post-traumatiques et des syringomyélies [3, I 2]. La substitution TDM-IRM sera dans ce domaine, complète et rapide. Il en sera probablement de même pour le bilan d'extension des tumeurs du massif facial, où l'IRM semble capable de faire la part entre l'infiltration tumorale et les lésions inflammatoires associées, et pour le bilan des tumeurs laryngées où l'IRM démontre une meilleure définition de l'extension dans les parties molles.

\section{Au niveau du thorax}

L'IRM bénéficie de l'imagerie longitudinale et du contraste spontané offert par les vaisseaux du médiastin dont la lumière restée vide de signal, en raison des phénomènes de flux, est facilement repérée. Certes, la résolution spatiale de l'IRM demeure inférieure à celle de la TDM mais ceci est largement compensé par l'excellente résolution en contraste, en particulier bien perçue au niveau médiastinal et hilaire [13].

Malgré ces avantages théoriques, les premières études comparatives entre TDM et IRM ne démontrent pas de différence significative en termes de sensibilité et de spécificité, en particulier pour le diagnostic d'extension locorégionale des cancers bronchopulmonaires opérables [i4]. L'IRM comme la TDM se heurte au manque de spécificité des lésions hilaires et médiastinales. La différenciation entre adénopathies inflammatoires et adénopathies tumorales [ $\mathrm{I}_{5}$ ] n'est pas réalisable de manière fiable et reproductible (figure 4, p. 135). En ce qui concerne les affections pulmonaires, détection de nodules parenchymateux, diagnostic et bilan de bronchectasies, d'emphysème ou de pneumopathie focale et diffuse, la TDM reste encore indiscutablement supérieure à l'IRM [16].

\section{L'exploration du ceur et des gros vaisseaux}

Elle est rendue possible par la synchronisation du signal avec l'ECG. Des séquences dynamiques avec acquisition de coupes rapides permettent de reproduire une notion cinétique. Les possibilités de l'IRM ont peut-être été partiellement évaluées dans le diagnostic d'ischémie myocardique récente et chronique, les cardiomyopathies obstructives et les cardiopathies congénitales [17]. On ne peut pas parler dans ce domaine de substitution TDM-IRM, tant la place de la TDM est réduite dans l'exploration cardiaque. Toutefois, une concurrence se dessine dans un proche avenir entre l'IRM et le " ciné CT", qui est un nouveau scanographe à acquisition dynamique permettant des études cinétiques spécifiquement adaptées aux cavités cardiaques [ 17 ].

Il est encore difficile de préciser la place de l'IRM entre, d'une part l'échographie bidimensionnelle, dont la sensibilité est proche de celle de l'IRM pour un rapport coût-efficacité comparativement supérieur et, d'autre part les cathétérismes cardiaques qui sont encore souvent nécessaires dans les bilans pré-opératoires. L'avenir réel du développement de l'IRM dans le domaine cardiovasculaire dépend encore largement des progrès technologiques qui surviendront dans les mois ou années à venir. Dans l'exploration des affections de l'aorte, anévrysmes, dissections, coarctations [1 8- 


\section{RÉFÉRENCES}

17. Higgins CB. New horizons in cardiac imaging. Radiology 1985; 156: 577-88.

18. Amparo EG, Higgins CB, Hricak $\mathrm{H}$, et al Aortic dissection: magnetic resonance imaging. Radiology 1985; 155 : 399-406.

19. Amparo EG, Hoddick WK, Hricak $\mathrm{H}$, et al. Comparison of magnetic resonance imaging and ultrasonography in the evaluation of abdominal aortic anevrysms. Radiology $1985 ; 154: 451-6$

20. Amparo EG, Higgins CB, Shafton EP. Demonstration of coartactation of the aorta by magnetic resonance imaging. $A 7 R$ 1984; $143: 1192-$ 4.

21. Stark DD, Moss AA, Goldberg HI, et al. Magnetic resonance and computed tomography of the normal and diseased pancreas: a comparative study. Radiology 1984; 150 : 153-62.

22. Heiken JP, Lee JKT, Glazer HS, et al. Hepatic metastases studied with magnetic resonance and computed tomography. Radiology $1985 ; 156$ : 423-7.

23. Ohtomo K, Itai Y, Furui S, et al. Hepatic tumors: differentiation by transverse relaxation time (T2) of magnetic resonance imaging. Radiology $1985 ; 155: 421-3$.

24. Glazer GM, Aisen AM, Francis JR, et al. Hepatic cavernous hemangioma: magnetic resonance imaging. Radiology $1985 ; 155: 417-20$.

25. Schultz CL, Haaga JR, Fletcher BD, et al Magnetic resonance imaging of the adrenal glands: a comparison with computed tomography. AfR 1984; 143 : $1235-40$.

26. Leung AWL, Bydder GM, Steiner RE, et al. Magnetic resonance imaging of the kidneys. $A F R$ 1984; 143 : 1215-27.

27. Butler H, Bryan PJ, Lipuma JP, et al. Magnetic resonance imaging of the abnormal female pelvis. $A 7 R$ 1984; 143: 1259-66.

28. Buonocore E, Hesemann C, Pavlicek W. Clinical and in vitro magnetic resonance imaging of prostatic carcinoma. $A 7 R$ 1984; 143: 1267-72.

29. McSweeney MB, Small WC, Cerny V, et al. Magnetic resonance imaging in the diagnosis of breast disease use of transverse relaxation times. Radiology 1984; 153: 741-4.

30. Edelman RR, Shoukimas GM, Stark DD, et al. High-resolution surface-coil imaging of lumbar disk discasc. $A 7 R$ 1985; $144: 1123-9$.

31. Paushter DM, Modic MT, Masaryk TJ. Magnetic resonance imaging of the spine: applications and limitations. Radiol Clin North Am 1985; 23: 551-62.

32. Fletcher BD, Scoles PV, Nelson AD. Osteomyelitis in children: detection by magnetic re-
20], une substitution TDM-IRM pourrait dès aujourd'hui se concevoir (figure 5).

\section{Au niveau abdominal}

La rate n'a pas encore été évaluée. Les résultats au niveau du pancréas sont extrêmement décevants [2I]. Les applications de l'IRM se résument surtout à ce jour à l'étude des tumeurs du foie. Dans ce domaine, la sensibilité de l'IRM semble voisine de celle de la TDM [22] mais la caractérisation reste extrêmement décevante [23] en dehors des angiomes hépatiques dont la sémiologie est assez reproductible [24]. Les résultats dans le bilan des carcinomes hépatocellulaires semblent démontrer une légère supériorité de l'IRM dans l'individualisation d'une capsulc péritumorale et l'extension veineuse. L'utilisation de produit de contraste (Gadolinium DTPA) n'entraîne pas de renseignements supplémentaires par rapport à ceux de la TDM avec étude dynamique de la prise de contraste iodée. (Voir l'article de Yre's Menu, p.rz2).

\section{Rétropéritoine, pelvis et seins}

L'IRM n'a pas démontré de supériorité flagrante dans le diagnostic des tumeurs surrénaliennes [25], ni dans le diagnostic et le bilan d'extension des cancers du rein [26]. Dans l'étude du pelvis et des sein's, les résultats de l'IRM sont globalement décevants [27-29]. Cette déception relève avant tout de l'absence totale de caractérisation tissulaire. L'IRM apparaît à peine supérieure à la TDM dont les possibilités très limitées sont aujourd'hui bien connues, dans le bilan des cancers de la vessie, de la prostate, de l'utérus, des ovaires et du rectum. De plus, la possibilité de différenciation entre fibrose et carcinose dans la surveillance postthérapeutique ne semble pas actuellement possible.

\section{Dans le domaine ostéo-articulaire}

L'IRM est ici riche de potentialités; c'est en effet la seule méthode non invasive pouvant établir le diagnos- tic de dégénérescence discale du fait de la diminution du signal discal liée à une baisse de sa teneur en eau [30]. L'IRM visualise les hernies discales aussi bien que la TDM et certains auteurs considèrent déjà l'IRM comme une véritable alternative à la TDM et à la myélographie pour le diagnostic des hernies discales. La TDM reste supérieure dans l'analyse des structures osseuses mais les progrès permanents des antennes de surface augmentent rapidement les possibilités de l'IRM [3I]. D'une façon générale, celle-ci semble permettre un diagnostic très précoce des affections osseuses, en particulier des ostéomyélites de l'enfant grâce à sa capacité de détection des modifications de la moelle osseuse et de l'os spongieux [32]. L'IRM parait également très prometteuse dans le diagnostic précoce des nécroses osseuses et dans l'évaluation des modifications post-radiques [33], domaines où la TDM est peu fiable. Dans le domaine des tumeurs osscuses, malgré l'absence de caractérisation tissulaire, il semblerait qu'une relation existe entre l'agressivité de la tumeur et l'allongement du paramètre $\mathrm{T}_{2}$ de la relaxation. L'extension tumorale vers l'os spongieux et vers les parties molles est mieux appréciée par l'IRM que par la TDM [34].

Dans l'exploration des articulations, le rôle de la TDM est assez limité. L'IRM démontre dans ce domaine de grandes possibilités, surtout pour l'analyse anatomique fine des différentes structures cartilagineuses et ligamentaires [35].

La sensibilité diagnostique de l'IRM approcherait celle de l'arthrographie et on peut espérer à terme une réduction du nombre des explorations invasives arthrographiques et arthroscopiques.

\section{Conc/usion}

Quel que soit le domaine d'application, l'IRM offre un certain nombre d'avantages qui la différencient de la TDM. Elle est une méthode diagnostique non invasive et non irradiante qui permet une imagerie en coupes multidirectionnelles favorable aux études anatomiques et au bilan morphologique des tumeurs. $m / s^{o} 3$ vol. 2 mars 86 


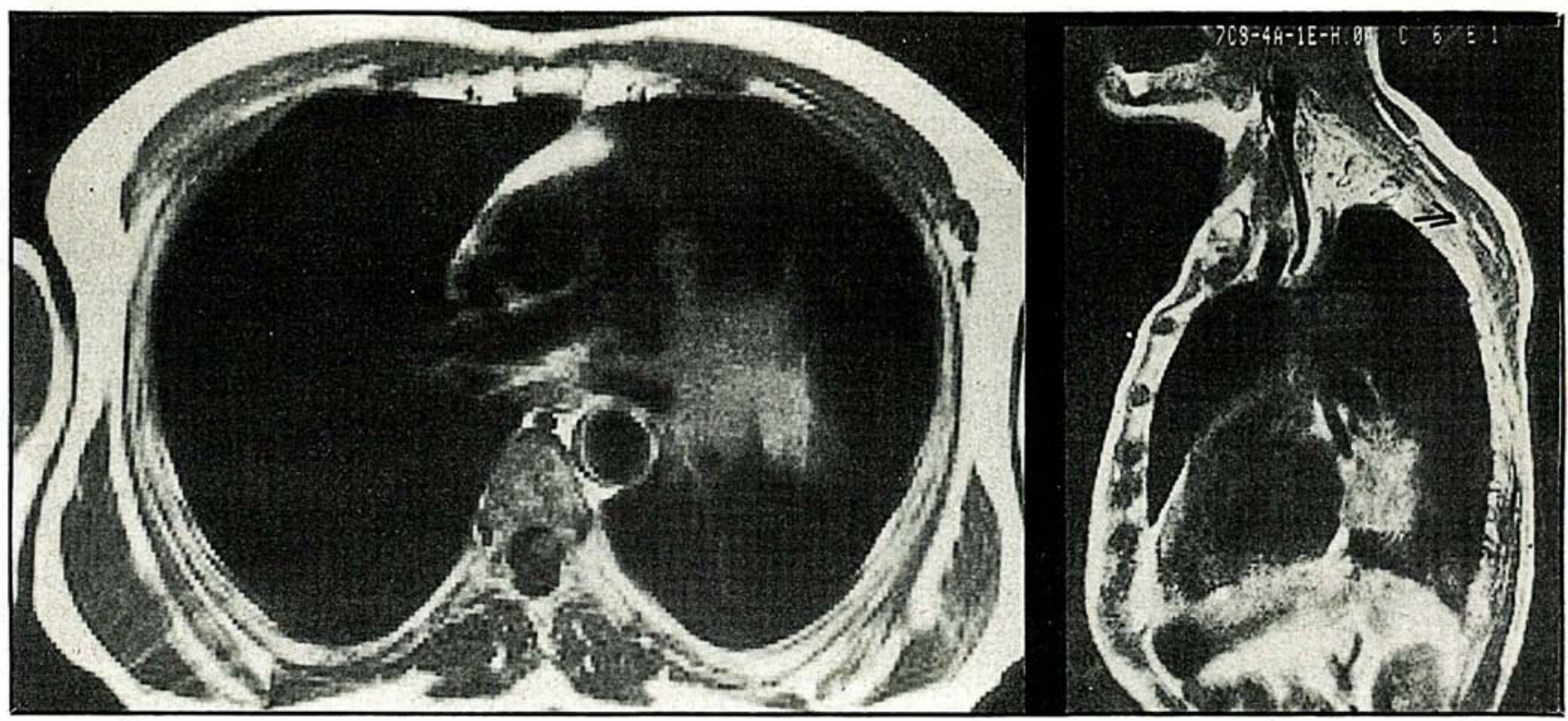

Figure 4. Cancer de la bronche lobaire inférieure.

Vue sagittale (à gauche) et vue axiale transverse (à droite).

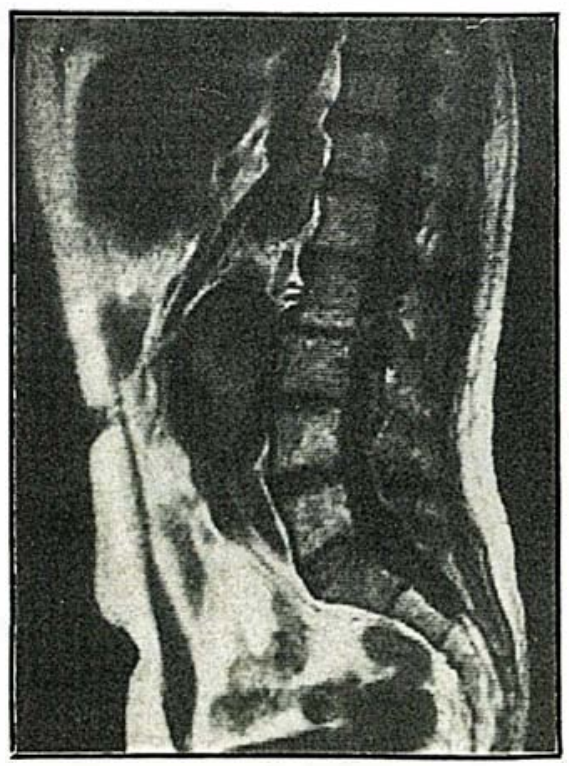

Figure 5. Anévrysme de l'aorte abdominale. Vue sagittale.
Elle offre une imagerie multiparamétrique dans laquelle interviennent non seulement la densité protonique, les temps de relaxation, mais aussi le flux, le décalage chimique et la diffusion. A ces avantages indiscutables, viennent s'opposer un certain nombre d'inconvénients qui limitent encore la diffusion de l'IRM : son coût reste élevé, compris entre deux et trois fois celui de la TDM; le temps d'examen reste long, compris entre une et deux heures, freinant la rentabilité de la méthode; la résolution spatiale est encore inférieure à celle de la TDM, surtout dans l'étude des organes rendus mobiles par la respiration. Mais la limite de l'IRM la plus décevante est certainement son échec dans la caractérisation tissulaire. Malgré les espoirs apportés par l'exploitation des paramètres de relaxation $\mathrm{T} 1$ et $\mathrm{T} 2$ dont les valeurs dépendent de l'environnement moléculaire des protons examinés, donc indirectement de la composition tissulaire, celle-ci reste insuffi- sante dans la plupart des cas pour éviter un prélèvement histologique de la lésion dépistée.

Toutefois, ces limites de l'IRM ne sont pas établies de manière définitive. Des perspectives d'amélioration peuvent être envisagées, dont certaines à court terme, telles que : l'amélioration des logiciels d'acquisition et d'exploitation qui permettra de raccourcir les temps d'examen; la mise au point de nouvelles méthodes d'excitation pour une "imagerie rapide", qui permettra de réduire les temps d'acquisition d'un facteur 3 à 5 ; la résolution spatiale qui sera améliorée par des logiciels de correction des artéfacts dus aux mouvements respiratoires, et le développement d'antennes de surface adaptées à toutes les régions examinables sur champ réduit : parties molles, articulations, rachis, moelle, cou, orbites, oreilles, seins, etc.; la caractérisation des lésions dépistées, qui pourra être améliorée par la mise au point et le développement d'agents de contraste modifiant la 
relaxation des tissus normaux et/ou des lésions. Les recherches dans ce domaine s'orientent d'une part vers des agents non spécifiques, le moins toxiques possible, d'autre part vers des agents spécifiques d'organes ou d'un type de lésion donnée. Le développement d'une imagerie de décalage chimique [36] ou d'une imagerie de diffusion [9] participera aussi à l'amélioration de la caractérisation tissulaire. L'imagerie de flux s'attachera à établir des mesures non invasives des débits cardiaque, artériel ou veineux [37]. La spectroscopie faite in vivo sur des échantillons tissulaires sélectionnés par l'imagerie est théoriquement faisable pour un certain nombre de noyaux mais se heurte encore à des contraintes technologiques non résolues [38].

De ce bilan réalisé à la fin de l'année 1985 , un certain nombre de points peuvent être relevés en termes de substitution et de complémentarité et en fonction des maladies étudiées.

- Une substitution de la TDM par l'IRM est possible à court terme dans certains domaines comme les - maladies de la moelle, les tumeurs de la fosse postérieure et du tronc cérébral.

- La TDM reste encore largement supérieure à l'IRM en pathologie pulmonaire et dans les affections du pancréas, du tube digestif et des mésos.

\section{RÉFÉRENCES}

33. Ramsey RG, Zacharias CE. Magnetic resonance imaging of the spine after radiation therapy: easily recognizable effects. $A 7 R \quad 1985 ; 144$ : I 13 - 5 .

34. Zimmer WD, Bcrquist TH, McLeod RA, et al. Bone tumors: magnctic resonance imaging versus computed tomography. Radiology 1985; 155: 709-18.

35. Rcicher MA, Bassett LW, Gold PH. Highresolution magnetic resonance imaging of the knce joint: pathologic correlations. $A \mathcal{F}$ 1985; I45: 903-9.

36. Lee JKT, Heiken JP, Dixon WT. Detection of hepatic mctastases by proton spectroscopic imaging. Radiology 1985; 156: 429-33.

37. Bradley WG, Waluch V. Blood flow: magnetic resonance imaging. Radiology 1985 ; 154 : 44350.

38. Maudsley AA, Hilal SK, Simon HE, et al. In vivo $M R$ spectroscopic imaging with $\mathrm{P}-3$ I. $R a$ stinivi $1984 ; 153$ : 745-50.
- L'IRM -semble devenir performante dans des domaines où le rôle de la TDM n'est que mineur et accessoire, comme l'exploration cardiovasculaire et la pathologie ostéoarticulaire.

- Il persiste enfin de nombreux domaines où les performances des deux examens n'apparaissent pas significativement différentes, comme les maladies des hémisphères cérébraux ainsi que les maladies médiastinales, hépatiques, rétropéritonéales et pelviennes.

C'est dans ce cadre que les études prospectives et comparatives, portant sur un grand nombre de malades, seront nécessaires pour définir les situations cliniques où une substitution d'un examen par l'autre pourrait être justifiée et celles où une stratégie entre les deux examens pourrait être utile

\section{Summary}

The current role of magnetic resonance imaging (MRI) and the eventuality of its substitution to computerized tomography (CT) are mostly unsolved problems because comparative studies are just beginning. Nevertheless, this article tries to compare the actual position of the diagnostic capabilities of MRI with those of $\mathrm{CT}$ in different pathologic fields. Technically, MR has several recognized advantages, particularly absence of ionizing radiation, lack of necessity for intravenous contrast material, multiplanar display and better contrast resolution. However, MRI is more expensive, examination times are longer and spatial resolution is limited. MRI has already established itself as the method of choice in the evaluation of various pathologic conditions of the posterior cerebral fossa and the spinal cord. In some other applications (intervertebral disk disease, bone tumors), MRI will probably soon demonstrate its diagnostic superiority on $\mathrm{CT}$. In numerous other fields, MRI and CT seem to be roughly equivalent (mediastinum, liver, retroperitoneum and pelvis). For lungs, pancreas and gastrointestinal tract, CT remains superior. For some organs where CT has a minor role, $M R$ is a very promising method, especially for heart and joint studies. However, it should be kept in mind that further investigations are needed to define precisely the respective role of these two methods and that the clinical efficacy of MRI will undoubtedly increase as the technology is refined.

\section{TIRÉS A PART}

P. Grenier : Servicc de radiologie, hôpital Beaujon, 100, boulevard du Général-Leclerc, 92118 Clichy Cedex. 